\title{
A Dynamical Systems Approach to Schwarzschild Null Geodesics
}

\author{
Edward Belbruno $^{1,2}$ and Frans Pretorius ${ }^{3}$ \\ ${ }^{1}$ Courant Institute of Mathematical Sciences, New York University \\ 2 Princeton University \\ ${ }^{3}$ Department of Physics, Princeton University
}

\begin{abstract}
The null geodesics of a Schwarzschild black hole are studied from a dynamical systems perspective. Written in terms of Kerr-Schild coordinates, the null geodesic equation takes on the simple form of a particle moving under the influence of a Newtonian central force with an inverse-cubic potential. We apply a McGehee transformation to these equations, which clearly elucidates the full phase space of solutions. All the null geodesics belong to one of four families of invariant manifolds and their limiting cases, further characterized by the angular momentum $L$ of the orbit: for $|L|>\left|L_{c}\right|$, (1) the set that flow outward from the white hole, turn around, then fall into the black hole, (2) the set that fall inward from past null infinity, turn around outside the black hole to continue to future null infinity, and for $|L|<\left|L_{c}\right|$, (3) the set that flow outward from the white hole and continue to future null infinity, (4) the set that flow inward from past null infinity and into the black hole. The critical angular momentum $L_{c}$ corresponds to the unstable circular orbit at $r=3 M$, and the homoclinic orbits associated with it. There are two additional critical points of the flow at the singularity at $r=0$. Though the solutions of geodesic motion and Hamiltonian flow we describe here are well known, what we believe is a novel aspect of this work is the mapping between the two equivalent descriptions, and the different insights each approach can give to the problem. For example, the McGehee picture points to a particularly interesting limiting case of the class (1) that move from the white to black hole: in the $L \rightarrow \infty$ limit, as described in Schwarzschild coordinates, these geodesics begin at $r=0$, flow along $t=$ constant lines, turn around at $r=2 M$, then continue to $r=0$. During this motion they circle in azimuth exactly once, and complete the journey in zero affine time.
\end{abstract}




\section{Introduction}

The Schwarzschild metric, describing a static, non-rotating black hole solution to the Einstein field equations, was discovered within a year of Einstein's publication of the theory of general relativity. Though it had to wait till the 1960's before the full nature of the metric was truly uncovered, it has nevertheless been well studied for nearly a century. One of the more important tools in this regard is understanding the geodesic structure of the spacetime; in particular, timelike and null geodesics characterize the paths that a freely moving test particle $P$ can follow within the spacetime. The geodesic equations of motion for $P$ are a set of second order, ordinary differential equations, describing the evolution of the coordinates of $P$ as a function of an affine parameter. In Schwarzschild these equations are integrable, and the solutions have been known for a long time (see any standard text on general relativity). Nevertheless, the solutions exhibit a sufficiently rich set of dynamics that new insights into them are still being garnered. For example, in the past couple of decades dynamical systems methods have been brought to bare on the geodesic equations, and perturbations thereof. A couple of the interesting results have been a new taxonomy of orbits based on the subset of orbits that are periodic, emphasizing the importance of the homoclinic orbits that asymptotically approach the unstable branch of circular orbits [1, 2, 3, and that a generic perturbation of the geodesic flow possesses a chaotic invariant set [4, 5, 6, 7, 8, 9, 10,

In this paper we describe a new approach to understand the phase space of geodesic orbits, by applying a transformation due to McGehee [11]. This transformation is designed to resolve the singularities that formally appear in the Newtonian equations of motion when particles interacting through a central force collide. The transformation "blows up" collision into an invariant manifold for the flow of the transformed differential equations. This allows the motion of $P$ to be studied near collision, uncovering interesting dynamics. To apply these methods, we map the geodesic equation, written in Cartesian-like Kerr-Schild [12] (or ingoing Eddington-Finkelstein [13, 14]) coordinates, to a central force problem. This is trivial for the case where $P$ has zero mass (null geodesic), which we focus on in this paper, though the methods can be generalized.

The rest of the paper is laid out as follows. In Section 2 we introduce the Schwarzschild metric in the standard Schwarzschild coordinates, give an overview of its Penrose diagram, and qualitatively discuss the null geodesics of the geometry. This will provide a point of reference as we map the geometric picture to a dynamical systems description within McGehee coordinates. In Section 3 the differential equations for the motion of $P$ are given, in Schwarzschild and Kerr-Schild coordinates. In Section 4 we briefly summarize the results of the McGehee method for understanding the flow of a general class of Hamiltonian systems corresponding to central force problems. In Section 5 we restrict to the particular system that maps to the null geodesic structure of Schwarzschild. We show that the phase space flow can be subdivided into four families of invariant manifolds and their limiting cases. There are four critical points of the flow - the black hole and white hole singularities at $r=0$, and the two (with angular momentum $L= \pm L_{c}$ ) unstable circular orbits at $r=3 M$. Though much of the understanding of geodesic motion and the classical Hamiltonian system are individually well known, we believe the novel aspect of this work is the (sometimes non-trivial) mapping between the two equivalent descriptions, and the insight one gives to the other. One particularly interesting example is the limiting case of geodesics that 
in the dynamical systems description flow "directly" from the the white to black hole: the collective set of these geodesics trace out the interior region of the white/black hole, flow along Schwarzschild $t=$ const. lines, and complete exactly one orbit $\Delta \Phi=2 \pi$ along the journey from white hole to black hole singularity. Also, the standard affine parameter integrates to zero along these curves. To obtain a finite affine length requires rescaling it by an angular momentum dependent quantity, which diverges in the limit. Details of all this are discussed in Section 5 . Finally, we conclude in Section 6 with a summary, and discussion of possible future extensions and applications. Throughout we use geometric units, where the speed of light $c=1$ and Newton's constant $G=1$.

\section{The Schwarzschild Geometry}

The Schwarzschild metric, describing a non-rotating black hole of mass $M$, has the following line element in standard (spherical polar) Schwarzschild coordinates:

$$
d s^{2}=g_{\alpha \beta} d x^{\alpha} d x^{\beta}=-(1-2 M / r) d t^{2}+(1-2 M / r)^{-1} d r^{2}+r^{2}\left(d \theta^{2}+\sin ^{2} \theta d \phi^{2}\right),
$$

where $g_{\alpha \beta}$ is the metric tensor, and we use the Einstein summation convention where repeat indices are summed over. Figure 1 shows a Penrose diagram of the Schwarzschild spacetime. For readers not familiar with this diagram, we will briefly review its salient features. The Penrose diagram is a conformal compactification of the maximal analytic extension of the Schwarzschild metric, designed to highlight the causal structure of the spacetime. Here we project out the $(\theta, \phi)$ coordinates, so a point on the diagram represents a two-sphere of area $4 \pi r^{2}$. Radial (zero angular momentum) null geodesics are straight lines angled at $\pm 45^{\circ}$ relative to the horizontal. Any causal curve that could be associated with a particle trajectory (whether timelike, null, geodesic or not) has a slope $\geq 45^{\circ}$ everywhere along its projected curve on the diagram. There are two identical, causally disconnected, asymptotically flat regions reached as $r \rightarrow \infty$; without loss of generality we focus on the right hand region on the diagram. Similarly, there are two singularities in the geometry at $r=0$ : the white hole singularity occurring to the past of any event in the spacetime, and the black hole singularity that is within the causal future of any event.

A null curved can be parameterized by an affine time $\sigma$, unique up to a constant scaling and translation. Null curves that originate at $r=\infty$ (with $\sigma \rightarrow-\infty$ ) come from a region called past null infinity $\Im^{-}$, and those that return to $r=\infty$ (as $\left.\sigma \rightarrow+\infty\right)$ end at future null infinity $\Im^{+}$. The event horizon of the black hole is formally defined as the boundary of the causal past of $\Im^{+}$, which on the Penrose diagram is the line labeled $r=2 M, t=\infty$. Null curves that come from the white hole "begin" at $r=0$ with finite $\sigma$, and those that cross the event horizon reach $r=0$ in finite affine time. Note that the Schwarzschild time coordinate $t$ is singular at $r=2 M$. Further, note that $r$ and $t$ switch "character" at $r=2 M$ : outside, $r$ is spacelike, $t$ timelike, and vice-versa inside. In the black hole, time as measured by $r$ flows to smaller $r$ (the opposite in the white hole). Thus, the inevitability of encountering the singular at $r=0$ for any observer crossing the horizon is evident in the diagram: $r=0$ is no longer a "place" that can be avoided, rather it is a "time" that will happen for any such observer. Finally, note that the compactification severely distorts the spacetime at the corner points of the diagram - that $r=0, r=2 M$ and $r=\infty$ touch in the diagram is purely an artifact of the compactification. 


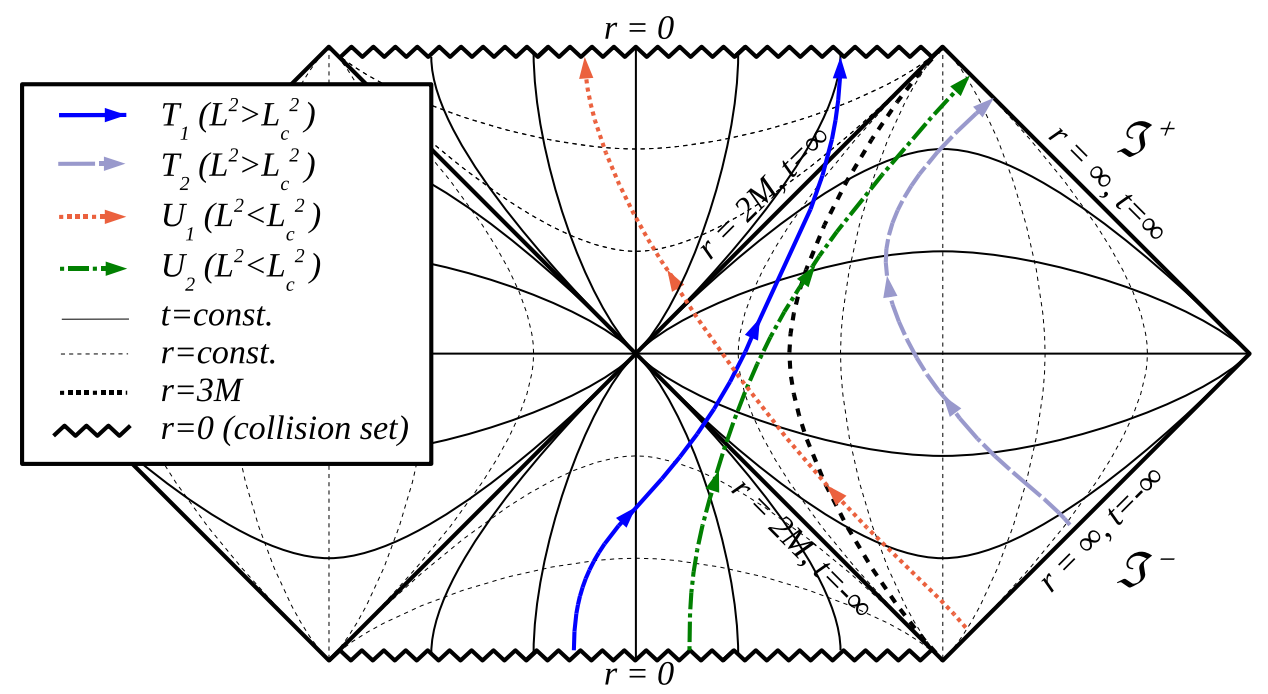

Figure 1. A schematic Penrose diagram of the Schwarzschild solution, with representative null geodesics from each of the invariant manifolds; see Figure 2 for the corresponding diagram in McGehee coordinates.

\section{Geodesic Equations in the Schwarzschild Spacetime}

We begin by describing the geodesic equations for causal particles; later we restrict to the null case. The geodesic equation for a particle $P$ in parametric form $x^{\alpha}(\sigma)$ is

$$
\ddot{x}^{\alpha}+\Gamma_{\beta \gamma}^{\alpha} \dot{x}^{\beta} \dot{x}^{\gamma}=0
$$

where the over-dot ${ }^{\circ} \equiv d / d \sigma$ and $\Gamma_{\beta \gamma}^{\alpha}$ is the metric connection. This is formally a set of second order ordinary differential equations, though due to the symmetries of the Schwarzschild metric, and the normalization condition that $g_{\alpha \beta} \dot{x}^{\alpha} \dot{x}^{\beta}$ equals $0(-1)$ for null (timelike) geodesics, one obtains the following first integrals of motion of (2) in Schwarzschild coordinates (1):

$$
\begin{aligned}
& \dot{t}=\tilde{E}(1-2 M / r)^{-1} \\
& r^{4} \dot{r}^{2}=\tilde{E}^{2} r^{4}-\left(r^{2}-2 M r\right)\left(\mu^{2} r^{2}+K\right) \\
& r^{4} \dot{\theta}^{2}=K-L^{2} \sin ^{-2} \theta \\
& \dot{\phi}=L\left(r^{2} \sin ^{2} \theta\right)^{-1},
\end{aligned}
$$

where $\mu^{2}$ is the rest mass of $P, \tilde{E}$ its energy, $L$ its angular momentum about the axis $\sin \theta=0$, and $K$ is Carter's constant of motion. Note that the second equation above for $\dot{r}$ can be written as a Newtonian central force problem

$$
E=\frac{1}{2} \dot{r}^{2}+V(r),
$$

with equation of motion

$$
\ddot{r}=-d V / d r,
$$


where $E=\tilde{E}^{2} / 2$ and the effective potential $V(r)$ is given by

$$
V(r)=\frac{1}{2}\left(1-\frac{2 M}{r}\right)\left(\mu^{2}+\frac{K}{r^{2}}\right) .
$$

\section{Kerr-Schild coordinates}

The Schwarzschild metric in Cartesian Kerr-Schild coordinates $(\lambda, x, y, z)$ is

$$
d s^{2}=-d \lambda^{2}+d x^{2}+d y^{2}+d z^{2}+2 M r^{-3}(x d x+y d y+z d z+r d \lambda)^{2},
$$

related to Schwarzschild coordinates by the set of transformations

$$
x=r \sin \theta \cos \phi, \quad y=r \sin \theta \sin \phi, \quad z=r \cos \theta
$$

and

$$
d \lambda=d t+\frac{2 M}{r-2 M} d r .
$$

One advantage of Kerr-Schild coordinates over Schwarzschild coordinates, as is evident from (17), is that they are regular across the event horizon at $r=2 M$.

The geodesic equations in Kerr-Schild coordinates can be written as [15]

$$
\begin{aligned}
& \dot{\lambda}=2 M K r^{-3}(\tilde{E}-\dot{r})^{-1}+\tilde{E} \\
& \ddot{x}_{k}=-3 M K x_{k} r^{-5}-\mu^{2} M x_{k} r^{-3},
\end{aligned}
$$

where for the sake of notation, $k=1,2,3$, with $x_{1}=x, x_{2}=y, x_{3}=z$. Due to the spherical symmetry of the Schwarzschild spacetime, without loss of generality we can restrict attention to planar motion. For simplicity we choose $z=0$, corresponding to $\theta=\pi / 2$, for which $K=L^{2}(3)$. In the expressions below we will replace $K$ with $L^{2}$, though one can consider them to be valid for motion in any plane if $L$ is re-interpreted as the angular momentum relative to an axis orthogonal to the orbital plane. We will also now limit the discussion to null geodesics, for which $\mu^{2}=0$, and only focus on the coordinate flow (11) of the geodesics:

$$
\ddot{\mathbf{x}}=-3 M L^{2} \mathbf{x} r^{-5},
$$

where $\mathbf{x}=\left(x_{1}, x_{2}\right) \in R^{2}$, and $r=|\mathbf{x}|=\sqrt{x_{1}^{2}+x_{2}^{2}}$.

The corresponding Hamiltonian for this system of equations is

$$
E=H(\mathbf{x}, \dot{\mathbf{x}})=\frac{1}{2}|\dot{\mathbf{x}}|^{2}-L^{2} M|\mathbf{x}|^{-3},
$$

where $E=\tilde{E}^{2} / 2$ is a positive constant of motion for any particle trajectory.

$\ddagger$ Technically, (7) is only regular across the black hole event horizon, and not the Cauchy horizon at $r=2 M$ of the white hole. The reason is $\lambda$ has been chosen so that along an $r=$ const. surface, $\lambda$ coincides with the retarded time $v$ of an ingoing radial null curve coming from $\Im^{-}$, and $v \rightarrow-\infty$ as the white hole horizon is approached on the Penrose diagram. These coordinates are thus also referred to as ingoing Eddington-Finkelstein coordinates. If instead we had chosen a coordinate $\bar{\lambda}=d t-2 M /(r-2 M) d r$, then $\bar{\lambda}$ would coincide with advanced time $u$ of an outgoing radial photon along an $r=$ const. surface (outgoing Eddington-Finkelstein coordinates), and the metric would be regular at the white hole horizon, but singular at the black hole horizon for similar reasons. However, at the end of the day one arrives at the same geodesic equation of interest (12) for the spatial Cartesian coordinates as a function of affine time $[x(\sigma), y(\sigma), z(\sigma)]$, which is well defined across both horizons. 
It is noted that for each value $h \geq 0$ of the energy $E$, the motion of $P$ of mass zero lies on the three-dimensional energy surface

$$
\Sigma=\left\{(\mathbf{x}, \dot{\mathbf{x}}) \in R^{4} \mid H(\mathbf{x})=h \geq 0\right\} .
$$

To facilitate direct application of prior work on Newtonian central force problems of the form (12), it is useful to perform the following dimensionful rescaling of the affine parameter $\sigma$ to $\xi$

$$
\sigma=\frac{\xi}{\sqrt{L^{2} M}} .
$$

This transforms (12) to

$$
\frac{d^{2} \mathbf{x}}{d \xi^{2}}=-3 \mathbf{x} r^{-5},
$$

In the following section we consider the solutions $\mathbf{x}(\xi)=\left(x_{1}(\xi), x_{2}(\xi)\right)$ of (16).

\section{Transformation to McGehee Coordinates and Blowup of Collision}

We consider general central force fields with potential

$$
U(\mathbf{x})=-|\mathbf{x}|^{-\alpha},
$$

$\alpha>0$, later restricting to the case $\alpha=3$ of (16). The system of differential equation describing the motion of a single particle with this potential is given by

$$
\ddot{\mathbf{x}}=-\partial_{\mathbf{x}} U(\mathbf{x})=-\alpha|\mathbf{x}|^{-\alpha-2} \mathbf{x} .
$$

It is convenient to write this as the first order system

$$
\begin{aligned}
& \dot{\mathbf{x}}=\mathbf{y}, \\
& \dot{\mathbf{y}}=-\alpha|\mathbf{x}|^{-\alpha-2} \mathbf{x} .
\end{aligned}
$$

This is a Hamiltonian system with Hamiltonian function

$$
H(\mathbf{x}, \mathbf{y})=\frac{1}{2}|\mathbf{y}|^{2}-|\mathbf{x}|^{-\alpha},
$$

which is the total energy of the particle and is conserved along solutions of (19), where $(\mathbf{x}, \mathbf{y}) \in R^{4}$.

The general description of the orbit structure for (19) was carried out in [11, with a special emphasis on the motion of the particle near collision. The general approach taken is to find a change of coordinates which have the effect of blowing up the collision, corresponding to $\mathbf{x}=\mathbf{0}$, into an invariant manifold with its own flow. When this is done, the dynamics of the particle near collision can be completely understood and solutions tending towards collision are asymptotic to this manifold. This change of coordinates also gives the global flow for the differential equations.

Set $\mathbf{X}=(\mathbf{x}, \mathbf{y})$, and consider a solution $\mathbf{X}(\xi)=(\mathbf{x}(\xi), \mathbf{y}(\xi))$ for (19) with an initial condition $\mathbf{X}(0)$. The standard existence and uniqueness theorems of differential equations guarantee that $\mathbf{X}(\xi)$ can be uniquely determined and defined over a maximal interval $\left(\xi^{-}, \xi^{+}\right)$, where $-\infty \leq \xi^{-}<0<\xi^{+} \leq+\infty$. 
Definition If $\xi^{+}<\infty$, then $\mathbf{X}(\xi)$ ends in a singularity at $\xi^{+}$. If $\xi^{-} \geq-\infty$ then $\mathbf{X}(\xi)$ begins in a singularity at $\xi^{-}$. In either case, $\xi^{*}=\xi^{+}$or $\xi^{-}$is said to be a singularity of the solution $\mathbf{X}(\xi)$.

The following result is proven in [11:

Let $\mathbf{X}(\xi)$ be a solution of (19) with a singularity at $\xi^{*}$. Then this singularity is due to collision. That is, $\mathbf{x}(\xi) \rightarrow 0$ as $z \rightarrow \xi^{*}$.

It follows from (20) that for a collision solution, $|\mathbf{y}| \rightarrow \infty$ as $z \rightarrow \xi^{*}$. Hence, for (19) the only solutions that are singular are collision solutions, either ending or beginning in collision. There are several methods available to study collision, the dynamics of solutions near it and to understand if a collision solution can be extended through the collision state in a smooth fashion. Here we present a brief summary of blowing up collision for (19) for arbitrary $\alpha>0$, and then apply that to understand the global flow for the case of $\alpha=3$. The details are in [11].

We set $\beta=\frac{\alpha}{2}, \gamma=(1+\beta)^{-1}$ for sake of notation. Also, it is convenient to use complex coordinates and identify the real plane $R^{2}$ with the complex plane $C^{1}$. Then, we can consider $\mathrm{x}$ to be a vector in $R^{2}$ or a complex number in $C^{1}$. The McGehee coordinates are given by the transformation $T$ of $\left(x_{1}, x_{2}, y_{1}, y_{2}\right)$ to $(\tilde{r}>0, \tilde{\theta}, w, v)$,

$$
\begin{aligned}
& \mathbf{x}=\tilde{r}^{\gamma} e^{i \tilde{\theta}} \\
& \mathbf{y}=\tilde{r}^{-\beta \gamma}(v+i w) e^{i \tilde{\theta}}
\end{aligned}
$$

and a transformation of the affine variable $\xi$,

$$
d \xi=\tilde{r} d \tau
$$

The system (19) is transformed into

$$
\begin{aligned}
& \tilde{r}^{\prime}=(\beta+1) \tilde{r} v, \\
& \tilde{\theta}^{\prime}=w, \\
& w^{\prime}=(\beta-1) w v, \\
& v^{\prime}=w^{2}+\beta\left(v^{2}-2\right),
\end{aligned}
$$

where prime denotes differentiation wrt $\tau$. In complex notation, the angular momentum for (19) is given by

$$
\Omega(\mathbf{x}, \mathbf{y})=\Im(\overline{\mathbf{x}} \mathbf{y}) .
$$

We fix the energy $H$ to the constant value $h$ and $\Omega$ to the constant value $c$ (not to be confused with the speed of light). $T$ transforms $H=h$ and $\Omega=c$ into

$$
\begin{aligned}
& w^{2}+v^{2}-2=2 h \tilde{r}^{\alpha \gamma} \\
& \tilde{r}^{(1-\beta) \gamma} w=c .
\end{aligned}
$$

We define the constant energy manifold,

$$
\mathbf{M}(h)=\left\{(\tilde{r}, \tilde{\theta}, w, v) \in R^{4} \mid \tilde{r} \geq 0, H=h\right\} .
$$

We define the collision set corresponding to collisions for System (19) as

$$
\mathbf{N}=\{(\tilde{r}, \tilde{\theta}, w, v) \in \mathbf{M}(h) \mid \tilde{r}=0\} .
$$


On account of (25), (26) and (28) can be written as

$$
\begin{aligned}
& \mathbf{N}=\left\{(\tilde{r}, \tilde{\theta}, w, v) \in \mathbf{M}(h) \mid \tilde{r}=0, v^{2}=2, w=0\right\} \quad \beta>1, \\
& \mathbf{N}=\left\{(\tilde{r}, \tilde{\theta}, w, v) \in \mathbf{M}(h) \mid \tilde{r}=0, v^{2}+c^{2}=2, w=c\right\} \quad \beta=1, \\
& \mathbf{N}=\left\{(\tilde{r}, \tilde{\theta}, w, v) \in \mathbf{M}(h) \mid \tilde{r}=0, w^{2}+v^{2}=2, c=0\right\} \quad 0<\beta<1 .
\end{aligned}
$$

As is proven in [11,

$\mathbf{N}$ is an invariant manifold for the vector field defined by System (23). Collision orbits approach $\mathbf{N}$ asymptotically as $\tau \rightarrow \pm \infty$.

Definition $\quad \mathbf{N}$ is called a blow up of the collision $\tilde{r}=0$ on $\mathbf{M}(h)$.

It is also shown in [11] that System (23) reduces to the system consisting of the last two equations of (23),

$$
\begin{aligned}
& w^{\prime}=(\beta-1) w v, \\
& v^{\prime}=w^{2}+\beta\left(v^{2}-2\right) .
\end{aligned}
$$

This system has the integral

$$
\Lambda(w, v)=|w|^{\alpha}\left|v^{2}+w^{2}-2\right|^{1-\beta} .
$$

In summary, to understand the flow of (19) on $\mathbf{M}(h)$, we can use System (32), where the collision manifold is given by $\mathbf{N}$.

\section{McGehee Flow of Schwarzschild Null Geodesics}

We now consider the case of interest given by the Schwarzschild problem that we showed reduced to System (16). This corresponds to $\alpha=3, \beta=3 / 2, \gamma=2 / 5$. The flow for System (32) is depicted in Figure 2. Incidentally, this figure captures the qualitative behavior for all values of $\beta>1$. It turns out that the flows for $\beta \leq 1$ are drastically different, in particular the Kepler problem corresponds to $\beta=1 / 2$. Over the next several paragraphs we will describe the flow in some detail, though first it would be helpful to identify a few relations between the coordinates $(w, v)$ and physical coordinates, as well as establish the map between the constants of motion $(h, c)$ of solutions to System (19) and the physically relevant constants $L, \tilde{E}$ and $M$.

Relationships between constants and coordinates

Using (21) and (26), and the angular momentum of the geodesic in the physical picture $L=x \dot{y}-y \dot{x}$, one finds that the angular momentum $c$ of the System (19) evaluates to

$$
c= \pm \frac{1}{\sqrt{M}}
$$

This rather unusual relationship is due to the scaling (15) between the time parameters of the two descriptions. Likewise, it is straightforward to show that the energy $h$ of the System (19) relates to physical constants via

$$
h=\frac{\tilde{E}^{2}}{2 L^{2} M} .
$$

From (21), the relation between areal radius $r$ and $\tilde{r}$ for $\alpha=3$ is

$$
r=\tilde{r}^{2 / 5},
$$




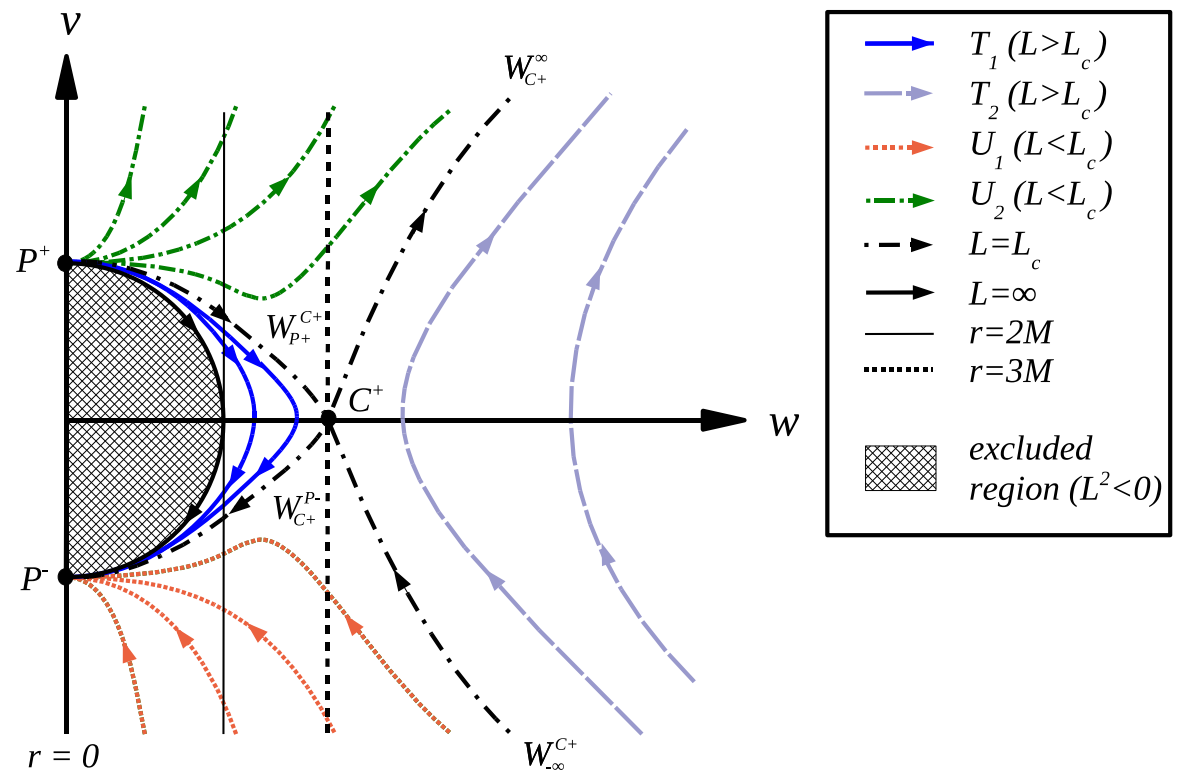

Figure 2. The Schwarzschild null geodesic flow in McGehee Coordinates; see Figure 1 for the corresponding Penrose diagram. The point $P^{-}\left(P^{+}\right)$here corresponds to the black hole (white hole) singularity at $r=0$. For clarity we only show the half plane $w>0$, corresponding to positive angular momentum $L$ orbits; the flow in $w<0$ for negative angular momentum orbits looks identical to that of $w>0$, but reflected about the $v$ axis.

and this together with (34) and (26) gives

$$
w= \pm \sqrt{\frac{r}{M}},
$$

where the plus (minus) sign corresponds to positive (negative) physical angular momentum $L$. Thus vertical lines of constant $w$ on the McGehee diagram correspond to surfaces of constant areal radius $r$. Putting all the above together, with (25), one finds that all trajectories projected onto the $(w, v)$ plane are characterized by the following polynomial with one free parameter $Q \geq 0$ :

$$
\begin{aligned}
& v^{2}=2-w^{2}+Q w^{6}, \\
& Q \equiv \frac{\tilde{E}^{2} M^{2}}{L^{2}} .
\end{aligned}
$$

Finally, note from (23) that $v=0$ implies $\tilde{r}^{\prime}=0$, which translates to $\dot{r}=0$; thus a trajectory that crosses $v=0$ corresponds to a geodesic that has a turning point in its radial motion.

\section{Particle Flow}

Figure 2 is a projection of the full flow in $(\tilde{r}, \tilde{\theta}, w, v)$ coordinates to the $(w, v)$ plane. Though as seen in (23), the two differential equations for $\left(w^{\prime}, v^{\prime}\right)$ do not depend on 
$(\tilde{r}, \tilde{\theta})$ and can be solved separately as an independent system. Once we know $(w, v)$ then the coordinates $(\tilde{r}, \tilde{\theta})$ can be easily computed for each $(w, v)$ by (23). ( $(\tilde{\theta}$ and $\tilde{r})$ represent polar-like coordinates for $P$, while $(w, v)$ can be viewed as velocity-like coordinates. System (23) implies that $\tilde{\theta}(\tau)$ either increases, $w>0$, or decreases $w<0$. This is just a cycling motion about the origin $\tilde{r}=0$ in position coordinates $\tilde{r}, \tilde{\theta}$. While this cycling motion is occurring, $\tilde{r}$ increases, $v>0$, or decreases, $v<0$. This is analogous to the projection on the Penrose diagram of the flow in $(r, t, \theta, \phi)$ to the (conformally compactified) $(r, t)$ plane, and cyclic motion for $K=L^{2}$ geodesics corresponds to increasing $\phi, L>0$, or decreasing $\phi, L<0$.

The flow curves $(w(\tau), v(\tau))$ in Figure 2 can be viewed as invariant manifolds. They foliate the $(w, v)$-plane. From (23) one can see that the flow has critical points $C^{ \pm}$at $(w, v)=( \pm \sqrt{3}, 0)$, where $\tilde{r}=\sqrt{3}$, and $\tilde{\theta}= \pm \sqrt{3} \tau$ (to within a constant phase) respectively. These correspond to the unstable (hyperbolic) circular periodic orbits at $r=3 M$. The periodic orbits $C^{ \pm}$exist for each $h>0$ on each energy surface $\mathbf{M}(h)$.

\section{Excluded region}

The flow on $\mathbf{M}(h)$ for (32) projects into the set $\left\{w^{2}+v^{2} \leq 2\right\}$ for each $h<0$, to $\left\{w^{2}+v^{2}=2\right\}$ for $h=0$, and to $\left\{w^{2}+v^{2}>2\right\}$ for $h>2$. Hence, since $P$ moves on $\Sigma$, defined by (14), where $h \geq 0$, then we need not consider the interior of the disc, $\left\{w^{2}+v^{2}<2\right\}$. So, in Figure 2 the region of interest are all points $\left\{w^{2}+v^{2} \geq 2\right\}$.

The collision set

The collision set $\mathbf{N}$ for $\alpha=3$ reduces to two critical points of the flow, $p^{+}=(0, \sqrt{2})$ and $p^{-}=(0,-\sqrt{2})$. These are unstable hyperbolic points. The flow tends to $p^{+}$as $\tau \rightarrow-\infty$ and to $p^{-}$as $\tau \rightarrow+\infty$. In the full phase space, these critical points have a constant value of $\tilde{\theta}$, and $\tilde{r}=0$. On the Penrose diagram, $p^{-}\left(p^{+}\right)$corresponds to the black hole (white hole) singularity at $r=0$.

Invariant manifolds of the flow

There are invariant manifolds connecting $C^{ \pm}$and $p^{ \pm}$. Two flow from $p^{+}$to $C^{ \pm}$and two flow from $C^{ \pm}$to $p^{-}$. These we label $W_{p^{+}}^{C^{ \pm}}$and $W_{C^{ \pm}}^{p^{-}}$, respectively; trajectories within this flow have physical angular momentum-squared $L_{c}^{2}=27 \tilde{E}^{2} M^{2}$. As $P$ moves along, e.g. $W_{p^{+}}^{C^{+}}$, it can be viewed in position space as cycling about the origin while moving outward toward the periodic orbit. The cycles converge to the periodic orbit asymptotically as $\tau \rightarrow \infty$ for $W_{p^{+}}^{C^{+}}$, and $\tau \rightarrow-\infty$ for $W_{C^{+}}^{p^{-}}$.

There are curves that leave $p^{+}$, move out to a maximum distance within the range $\sqrt{2}<w<\sqrt{3}(2 M<r<3 M)$ when $v=0$, then turn around and move to $p^{-}$. We label these manifolds $W_{p^{+}}^{p^{-}}$, and all of these geodesics have $L^{2}>L_{c}^{2}$. The union of these manifolds fill a region labeled $T_{1}$ in Figure 2 In the limit $L \rightarrow \pm\left|L_{c}\right|$, the solutions asymptote to the union of $W_{p^{+}}^{C^{ \pm}}$and $W_{C^{ \pm}}^{p^{-}}$. The $L^{2} \rightarrow \infty$ limit $(h \rightarrow 0)$ are a rather interesting class of geodesics, which we discuss in the following subsection.

Similarly, we have solutions that come in from negative infinity $\left(\Im^{-}\right)$for $v<0$, reach a minimum distance from the black hole in the range $w>\sqrt{3}(r>3 M)$ when $v=0$, then go back out to positive infinity $\left(\Im^{+}\right)$with $v>0$; they lie on invariant manifolds we call $W_{-\infty}^{+\infty}$, and are labeled $T_{2}$ in Figure 2 As with $T_{1}$, all these geodesics have angular momentum squared $L^{2}>L_{c}^{2}$. In the limit $L \rightarrow \pm\left|L_{c}\right|$, they asymptote to the union of the manifolds $W_{-\infty}^{C^{ \pm}}$and $W_{C^{ \pm}}^{\infty}$, the manifolds that connect $\Im^{-}$to $C^{ \pm}$ 
and $C^{ \pm}$to $\Im^{+}$respectively. The solutions on $W_{-\infty}^{C^{ \pm}}$asymptotically $(\tau \rightarrow \infty)$ approach the circular orbit as they spiral towards it from $\Im^{-}$, and those on $W_{C^{ \pm}}^{\infty}$ (beginning at $\tau=-\infty)$ asymptotically spiral away from the circular orbit to $\Im^{+}$.

The remaining two regions of the flow we call $U_{1}$ and $U_{2}$. $U_{1}$ consists of the manifolds $W_{-\infty}^{p-}$, which spiral in from $\Im^{-}$into the black hole. They do not encounter a turning point in $r$. These geodesics all have $L^{2}<L_{c}^{2}$, and in the limit $L \rightarrow \pm\left|L_{c}\right|$ asymptotically approach the union of manifolds $W_{-\infty}^{C^{ \pm}}$and $W_{C^{ \pm}}^{p-}$. Similarly, $U_{1}$ consists of the manifolds $W_{p+}^{\infty}$, which spiral out from the white hole to $\Im^{-}$, do not have a turning point in $r$, have $L^{2}<L_{c}^{2}$, and in the limit $L \rightarrow \pm\left|L_{c}\right|$ asymptote to the union of $W_{p+}^{C^{ \pm}}$and $W_{C^{ \pm}}^{\infty}$.

The $L^{2} \rightarrow \infty(h=0)$ limit

In region $T_{1}$, geodesics with $h \rightarrow 0$ correspond to the limit $\ell^{2} \equiv L^{2} / \tilde{E}^{2} \rightarrow \infty$. Fixing the physical energy $\tilde{E}$ to be finite, these geodesics have several curious propertie $[\hat{\xi}$, most easily deduced from the geodesic equations in Schwarzschild coordinates (3). First, for $r \leq 2 M$,

$$
\frac{d t}{d r}= \pm \frac{r^{3 / 2}}{\sqrt{r(2 M-r)^{2}+\ell^{2}(2 M-r)}}
$$

where the $+(-)$ sign corresponds to the part of the trajectory in the white (black) hole where $\dot{r}>0(\dot{r}<0)$. Taking the limit $\ell \rightarrow \infty$, one gets that these geodesics projected onto the $(r, t)$ plane in the Penrose diagram correspond to $t=$ constant lines (and recall that inside the horizon $t$ is a spacelike coordinate); i.e. they emanate from the white hole singularity, turn around at the intersection of the event and Cauchy horizons at $r=2 M$, then continue to the black hole singularity. Next, we calculate how much cycling motion in $\phi$ they execute. From the geodesic equations

$$
\frac{d \phi}{d r}= \pm \frac{\ell}{\sqrt{r^{4}+r \ell^{2}(2 M-r)}}
$$

again where $+(-)$ corresponds to the white (black) hole regions. Taking the limit $\ell \rightarrow \infty$, and integrating from $r=0$ to $2 M$ in the white hole and back from $r=2 M$ to 0 in the black hole gives $|\Delta \Phi|=2 \pi$ for the journey - these geodesics circle in azimuth exactly once going from the white to black hole singularity. Finally, we compute the total affine time $\Delta \sigma=\int d \sigma$ from

$$
\frac{d \sigma}{d r}= \pm \frac{r^{3 / 2}}{\tilde{E} \sqrt{r^{3}+\ell^{2}(2 M-r)}}
$$

For finite $\tilde{E}$, in the limit $\ell \rightarrow \infty, \Delta \sigma=0$ ! This rather bizarre result could be remedied by an infinite rescaling of $\sigma \rightarrow \sigma\left(\ell_{0} / \ell\right)$, where $\ell_{0}$ is some finite constant with dimension of length to make the scaling dimensionless. It is unclear what the physical significance such a rescaling is.

\section{Topology of the flow}

The solutions in the flow $T_{1}$ that spiral out from the white hole, turn around at $\sqrt{2} \leq w \leq \sqrt{3}$, and return to the black hole, trace out surfaces that are topologically

$\S$ in contrast to the $\ell^{2} \rightarrow \infty$ limit region of $T_{2}$, which simply correspond to geodesics that pass by the black hole with an infinite impact parameter 
equivalent to two cones smoothly joined together at $v=0$, with vertices anchored in the critical points at $p^{+}, p-$; see Figure 3 The flows $U_{1}$ coming from $\Im^{-}$into the black hole topologically form an (infinite length) open cone, with the open end at $\Im^{-}$, and the vertex at $p^{+}$; similarly for the flows in $U_{2}$. The geodesics in $T_{2}$ that come in from $\Im^{-}$and return to $\Im^{+}$lie on families of surfaces that are topologically equivalent to (infinite) cylinders.
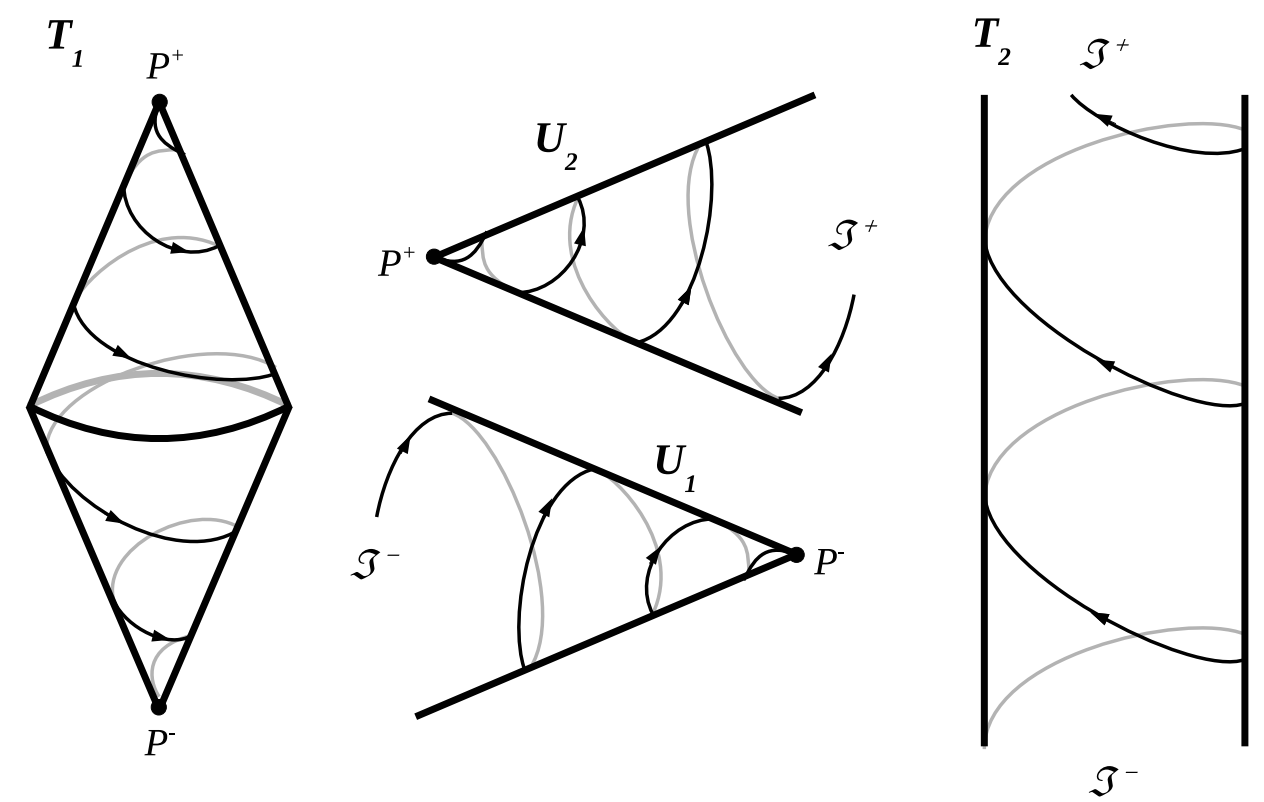

Figure 3. Topology of the flow traced out by solutions within each of the four invariant manifolds $T_{1}, T_{2}, U_{1}$ and $U_{2}$ (see Figure 21). Note that the sample trajectories are schematic only and for visual aid; only those solutions that flow close to the critical points $C^{ \pm}$trace out multiple cycles, and these are clustered near $w=\sqrt{3}(r=3 M)$.

\section{Extension of Solutions Through Collision}

Let $\mathbf{x}(\xi)$ be any solution of (18) which ends in collision at $\xi=0$. That is, $\mathbf{x}(\xi) \rightarrow 0$ as $\xi \rightarrow 0+$, where $\xi \rightarrow 0+$ means that $\xi$ approaches 0 where $\xi>0$. It is proven in [11] that $\mathbf{x}(\xi)$ is branch regularizable if and only if $\gamma \in \Xi$, where we define the set $\Xi$ as follows: Let $m, n$ be relatively prime integers, then $\Xi=\{m / n \mid n$ odd, $n>m>0\}$. Recall that $\gamma=(1+\beta)^{-1}, \alpha=2 \beta$. In our case, $\alpha=3$ and $\gamma=2 / 5$ belongs to the set $\Xi$.

We define branch regularizable as follows: A solution $\mathbf{x}=\mathbf{W}(\xi)$ of (18) which either begins or ends in collision at $\xi=\xi^{*}$ is branch regularizable at $\xi^{*}$ if it has a unique branch extension at $\xi^{*}$. A branch extension is defined by considering two solutions $\mathbf{W}_{\mathbf{1}}(\xi)$ and $\mathbf{W}_{\mathbf{2}}(\xi)$ of (18), where $\mathbf{W}_{\mathbf{1}}(\xi)$ ends in collision at $\xi^{*}$ and $\mathbf{W}_{\mathbf{2}}(\xi)$ begins in collision at $\xi^{*}$. Then, $\mathbf{W}_{\mathbf{2}}(\xi)$ is a branch extension of $\mathbf{W}_{\mathbf{1}}(\xi)$ if $\mathbf{W}_{\mathbf{2}}(\xi)$ is a real analytic continuation of $\mathbf{W}_{\mathbf{1}}(\xi)$ at $\xi=\xi^{*}$ for $\xi$ in a neighborhood of $\xi=\xi^{*}$. 
The fact that $\mathbf{x}(\xi)$ is branch regularizable implies that there is a way that solutions can smoothly and uniquely be extended through $p^{+}$in backwards time and through $p^{-}$in forwards time. For example, let $\mathbf{Z}(\xi)=(\mathbf{x}(\xi), \mathbf{y}(\xi))$ be a trajectory in phase space defined for $\xi<0$ that collides with $p^{-}$at $\xi=0$. Then there exists a unique extension of $\mathbf{Z}(\xi)$ for $\xi>0$. This extension is real analytic as a function of $\xi$ in a neighborhood of $\xi=0$, and corresponds to a smooth bounce of $P$ in $\left(x_{1}, x_{2}\right)$ space. In the spacetime picture, collision with $p^{-}$corresponds to the geodesic encountering the black hole singularity. The field equations of general relativity do not describe how spacetime can be extended beyond a singularity, and it is usually thought that a theory of quantum gravity is required to "resolve" the singularity. Nevertheless, one way to map the branch regularized extension to geodesic motion "through" the singularity would be to identify the black hole singularity with a white hole singularity of a second Schwarzschild solution of identical mas: $\llbracket$.

\section{Conclusions}

In this paper we have studied the relationship between the null geodesic structure of the Schwarzschild black hole solution, and the corresponding inverse-cubic Newtonian central force problem, using the methods of McGehee. Both these problems have been well studied before, though what we believe is novel in this paper is highlighting the exact correspondence between the two descriptions, allowing insights from the dynamical systems approach to be brought to the geodesic problem, and vice-versa. Indeed, in that regard it is rather amusing to note that McGehee titled his paper "Double Collisions for Classical Particle System with Nongravitational Interactions". It is also rather interesting that what in the Newtonian picture may be regarded as a clever "trick" using coordinate transformations to blow up the singular point of collision between two particles, is in a sense the natural way to describe Schwarzschild spacetime. Another remark is that understanding the invariant manifolds and unstable hyperbolic points allows standard techniques to be used to show that perturbations of the geodesic flow will generically cause chaotic motion. For example, for this purpose, solutions in $T_{1}$ may be regarded as forming a homoclinic loop if we identify $p^{+}$and $p^{-}$. Perturbations will then generically break the homoclinic loop and cause chaotic motion by the Smale-Birkhoff theorem [16].

Even though we restricted attention to null geodesics for simplicity, we expect that similar mappings could be used for timelike particles, or more complicated geometries like Kerr. This would be an interesting avenue for future work.

\section{Acknowledgements}

We would like to thank I. Rodnianski and D. Spergel for helpful discussions. This work was supported by the Alfred P. Sloan Foundation (FP), NSF grant PHY-0745779 (FP), and NASA/AISR grant NNX09AK61G (EB).

\section{References}

[1] J. Levin and G. Perez-Giz, Phys. Rev. D 77, 103005 (2008)

\| Identification with the white hole of the same solution is also a mathematical possibility, though that would create closed timelike curves within the spacetime, considered by some a class of "pathology" more severe than the black/white hole singularity. 
[2] J. Levin and G. Perez-Giz, Phys. Rev. D 79, 124013 (2009)

[3] G. Perez-Giz and J. Levin, Phys. Rev. D 79, 124014 (2009)

[4] R. Moeckel, Comm. Math. Phys., 150, 415 (1992)

[5] W. M. Vieira and P. S. Letelier, Phys. Rev. Lett. 76, 1409 (1996) [Erratum-ibid. 76, 4098 (1996)]

[6] P. S. Letelier and W. M. Vieira, Class. Quant. Grav. 14, 1249 (1997)

[7] N. J. Cornish and N. E. Frankel, Phys. Rev. D 56, 1903 (1997)

[8] S. Suzuki and K. i. Maeda, Phys. Rev. D 61, 024005 (2000)

[9] A. P. S. de Moura and P. S. Letelier, Phys. Rev. E 61, 6506 (2000)

[10] A. Saa and R. Venegeroles, Phys. Lett. A 259, 201 (1999)

[11] R. McGehee, Comment. Math. Helveti, 56 (1981), 524-557.

[12] R.P.Kerr and A. Schild, IV Centenario Della Nascita di Galileo Galilei, 222, (1964)

[13] A. S. Eddington, Nature 113 (1924)

[14] D. Finkelstein, Phys. Rev. 110, 965 (1958).

[15] J. A. Marck, Class. Quant. Grav 13 (1996), 393-402

[16] E. Belbruno, "Capture Dynamics and Chaotic Motions in Celestial Mechanics", Princeton University Press, 2004. 